Revista Aspas

ppgac - USP

Especial

\title{
AS ARTES CÊNICAS E AS POLÍTICAS PÚBLICAS DE CULTURA EM PONTE NOVA, MINAS GERAIS: UM BREVE RELATO DE EXPERIÊNCIA
}

THE PERFORMING ARTS AND PUBLIC CULTURE POLICIES IN PONTE NOVA, MINAS GERAIS: A BRIEF EXPERIENCE REPORT

\section{LAS POLÍTICAS DE ARTES ESCÉNICAS Y CULTURA PÚBLICA EN PONTE NOVA, MINAS GERAIS: UN BREVE INFORME DE EXPERIENCIA}

Emerson de Paula Silva e Luiz Gustavo Santos Cota

Professor do Curso de Teatro da UNIFAP - Universidade Federal do Amapá. Secretário Municipal de Cultura de Ponte Nova MG - 2013 a 2016.

Luiz Gustavo Santos Cota

Professor do Departamento de Fundamentos e Metodologias da Educação da UEMG - Universidade do Estado de Minas Gerais, Unidade Barbacena. Professor Permanente do Programa de Pós-Graduação, Mestrado Profissional, em Ensino de Ciências da Saúde e do Ambiente (PROCISA) da FADIP Faculdade Dinâmica do Vale do Piranga. 


\section{Resumo}

Este texto constitui um breve relato sobre as Políticas Públicas de Cultura na cidade de Ponte Nova - MG, abordando a implantação de ações como - Sistema Municipal de Financiamento à Cultura e os reflexos deste para a produção em Artes Cênicas na cidade. Busca-se também entender como os produtores teatrais dialogaram com o setor público de cultura local. Durante o relato busca-se também entender a constituição de uma política pública de cultura a nível nacional e como esta proposta pode dialogar com a realidade dos diferentes municípios brasileiros.

Palavras-chaves: Artes Cênicas, Políticas Públicas de Cultura, Ponte Nova.

\section{Abstract}

This text is a brief report about the Public Policies of Culture in the city of Ponte Nova - MG addressing the implementation of actions such as the Municipal Culture Financing System and its reflection for the production of Performing Arts in the city. It also seeks to understand how the theater producers dialogued with the public sector of local culture. During the report we also seek to understand the constitution of a public cultural policy at national level and how this proposal can dialogue with the reality of different Brazilian municipalities.

Keywords: Performing Arts, Public Culture Policies, Ponte Nova.

\section{Resumen}

Este texto es un breve informe sobre las Políticas Públicas de Cultura en la ciudad de Ponte Nova - MG que aborda la implementación de acciones como el Sistema Municipal de Financiamiento de la Cultura y su reflexión para la producción de Artes Escénicas en la ciudad. También busca comprender cómo los productores de teatro dialogaron con el sector público de la cultura local. Durante el informe también buscamos comprender la constitución de una política cultural pública a nivel nacional y cómo esta propuesta puede dialogar con la realidad de los diferentes municipios brasileños.

Palabras clave: Artes escénicas, Políticas de Cultura Pública, Ponte Nova. 
Durante o período de 2013 a 2016, a Secretaria Municipal de Cultura e Turismo de Ponte Nova, cidade pertencente a Zona da Mata Mineira, promoveu ações para a efetivação de uma Política Pública de Cultura na cidade, visando não só fortalecer a gestão pública de cultura no Município, bem como colocar a cidade em sintonia com um arranjo cultural que estimulasse a produção e profissionalização da Cultura local.

Um dos pontos chave do processo de implantação destas políticas culturais foi a criação do Sistema Municipal de Financiamento à Cultura que, através de um edital público, subsidiava a promoção, criação e difusão artística de grupos, artistas e coletivos ponte-novenses em suas diversas áreas de atuação fazendo com que os próprios agentes culturais pudessem deliberar sobre o uso e direcionamento do orçamento público para a Cultura na cidade.

Ponte Nova é uma cidade que possui reconhecida importância na Zona da Mata mineira, especialmente no que tange ao aspecto econômico macrorregional, mas também cultural. Uma das áreas artísticas de destaque são as Artes Cênicas, com importantes grupos de Teatro e Dança que movimentam a cidade, inclusive com produção de Festivais nestas áreas e que possuem repercussão estadual.

O Festival de Teatro de Ponte Nova (FESTPON) surgiu em 2010, fruto de uma ação conjunta dos grupos teatrais NAED (Núcleo Artístico Educacional), Cia Teatro de Bolso e Grupo de Teatro Viver com Arte, chegando em 2019 à sua 6ª edição. Tal Festival contou com aprovação pelo edital da Lei Estadual de Incentivo à Cultura do Estado de Minas Gerais, demonstrando a consolidação do projeto que fundamenta o FESTPON e do próprio Teatro no município. A produção cultural dos grupos citados fortaleceu um diálogo positivo e propositivo junto à política pública de cultura pontenovense, seja buscando o fortalecimento do referido Festival, seja nas produções específicas de cada grupo e/ou artista de Teatro da cidade, principalmente a partir da implantação do Sistema Municipal de Financiamento à Cultura.

Nesta perspectiva, antes de focar o relato da participação dos grupos teatrais neste processo, entendemos que se faz necessário refletir um pouco 
sobre o estabelecimento da Política Nacional de Cultura e como a mesma se estabelece no País através do estímulo à movimentação cultural nos municípios.

\section{Sobre os avanços das políticas e direitos culturais coletivamente construídos no Brasil, especialmente desde a Constituição Cidadã de 1988.}

Em seus artigos 215 e 216, o texto constitucional desenhou contornos mais claros em relação à Cultura, definida como um direito e um bem coletivo indispensável para a compreensão da dinâmica e complexa identidade nacional, determinando o livre acesso aos direitos culturais e a proteção do patrimônio cultural. Já seu artigo 216-A, fruto da emenda constitucional no.71, de 2012, definiu um passo à frente em relação às determinações já apresentadas preteritamente, com a criação do Sistema Nacional de Cultura (SNC), um dos frutos mais maduros das discussões e avanços das políticas públicas para o setor, nascidas do ventre constitucional e dos movimentos e agentes culturais brasileiros. ${ }^{1}$

Formal e burocraticamente embaralhados nas estruturas de outras áreas desde a década de 1950, especialmente da educação, os órgãos gestores da cultura apenas iniciaram seu processo de autonomia política e orçamentária em 1985, no governo Sarney, com a criação do Ministério da Cultura (MinC), o que possibilitou, durante um período que se estendeu até muito recentemente, um constante e estrutural aprimoramento e avanço das políticas culturais no país, representadas em ações de promoção, proteção, incentivo e financiamento, que tiveram na existência de um órgão específico e com orçamento próprio uma das principais forças motrizes. Não obstante $\circ$ processo de fortalecimento do setor, o MinC foi alvo de pelo menos três ações desarticuladoras, tendo sido a primeira no governo Collor (1990-1992); a segunda no governo Temer (2016-2018), quando houve intensa e consciente

\footnotetext{
'BRASIL. Constituição da República Federativa do Brasil de 1988. Disponível em: http://www.planalto.gov.br/ccivil 03/constituicao/constituicao.htm. Acesso em: 09 de janeiro de 2017.
} 
pressão da sociedade e agentes culturais contra extinção do órgão; e a terceira e atual ação desarticuladora, implementada pelo governo Bolsonaro, ainda em janeiro de 2019, quando o MinC foi extinto, sendo a gestão nacional de cultura incorporada à estrutura do recém-criado Ministério da Cidadania, na forma de uma Secretaria Especial da Cultura. Não sem razão, pairam incertezas e pessimismo quanto aos efeitos desarticuladores do rebaixamento político sofrido pela gestão máxima das políticas públicas de cultura para sua condição anterior à redemocratização do país.

Especialmente nos últimos vinte anos, a expansão de políticas e programas da área, para além do âmbito federal, fizeram com que estruturas administrativas, técnicas e políticas fossem transformadas ou engendradas em estados e municípios. Rodrigo Manoel Dias, professor da Universidade Federal da Fronteira Sul (UFFS), ao analisar estudos sobre as políticas culturais desenvolvidas no Brasil nas duas últimas décadas, afirma que a institucionalização de políticas públicas e a proliferação de ações desenvolvidas por agentes culturais consolidaram a cultura como item indispensável à vida coletiva e à própria pauta política, possibilitando a interiorização de iniciativas e projetos culturais, bem como a valorização e proteção estruturadas de nossas múltiplas formas de criar, fazer e viver. Ainda de acordo com o mesmo professor, tais transformações tiveram impacto direto nos municípios brasileiros, reorientando as ações dos atores públicos, redundando na criação de secretarias municipais de cultura e na atualização de fundações "para ajustar-se a modelos normativos e operativos nacionais". Tais mudanças impulsionaram a profissionalização dos agentes políticos e culturais, especialmente em produção cultural ou em gestão política da cultura, possibilitando que as mudanças que ocorriam em âmbito nacional (e global) atribuíssem à cultura um "espaço estratégico nas propostas de desenvolvimento, de revitalização das urbanidades e de outras formas de consumo de bens culturais". ${ }^{2}$

A capilarização dos órgãos de gestão da cultura, ou seja, das secretarias municipais de cultura, também foi objeto de análise da

\footnotetext{
${ }^{2}$ Idem, ibidem.
} 
pesquisadora Luana Vilutis, Doutora em Cultura e Sociedade pela Universidade Federal da Bahia (UFBA), que observou a maneira como a criação de secretarias avançou consideravelmente em tempos recentes. Em capítulo que integra o livro "Políticas culturais: olhares e contextos", publicado em 2015, a pesquisadora informa:

\begin{abstract}
Pesquisas revelam que mais de dois terços das secretarias exclusivas de cultura foram criadas na última década. Em 2006, apenas $4,2 \%$ dos municípios brasileiros possuíam uma secretaria exclusiva de cultura. Em 2012 esse dado triplicou, alcançando a média nacional de 13,5\%. Nos municípios entre 100 mil e 500 mil habitantes, a porcentagem de secretarias exclusivas aumenta para $36 \%$, o que revela a grande diferença entre os municípios pequenos e a média nacional. É interessante notar que houve um recuo na quantidade de municípios com fundação pública à frente do órgão gestor da cultura. Em 2006, esse dado correspondia a 2,6\% dos municípios e, em 2012, chegou a 2,1\% (IBGE, 2013). ${ }^{3}$
\end{abstract}

Luana Vilutis afirma que o aumento do número de municípios com secretaria exclusiva de cultura é um claro indicativo do avanço da institucionalização das políticas culturais brasileiras. A pesquisadora salienta, no entanto, que tão importante quanto a criação de secretarias municipais é a garantia da existência de orçamento próprio para a área e de que tais órgãos sejam ocupados por corpo técnico qualificado, sendo por ela consideradas questões de "necessidade significativa, urgente e atual". ${ }^{4}$

O Plano Nacional de Cultura (PCN), instituído pela Lei no..12.343, de 02 de dezembro de 2010, a partir de discussões nas quais se reuniram gestores e agentes culturais de todo país, e que se apresenta como o mais abrangente planejamento de políticas públicas de cultura no país, incluiu entre suas estratégias a expansão das secretarias municipais de cultura como parte do fortalecimento da gestão das políticas públicas do setor, para um prazo de dez anos, como apresentado no documento:

\footnotetext{
${ }^{3}$ VILUTIS, Luana. Pontos de Cultura e Planos Municipais: perspectivas da cooperação federativa. In: CALABRE, Lia. Políticas culturais: olhares e contextos. Rio de Janeiro: Fundação Casa de Rui Barbosa; São Paulo: Itaú Cultural, 2015, p.121. Disponível em:

http://d3nv1jy4u7zmsc.cloudfront.net/wp-content/uploads/2015/05/PoliticasCulturais02 v07.pdf. Acesso em: 08 de janeiro de 2017.

${ }^{4}$ Idem, ibidem.
} 


\title{
ESTRATÉGIAS E AÇÕES
}

(...)

1.1.6 Estimular a criação e instalação de secretarias municipais e estaduais de cultura em todo o território nacional, garantindo o atendimento das demandas dos cidadãos e a proteção dos bens e valores culturais. ${ }^{5}$

Através da Portaria n‥123, de 13 de dezembro de 2011, o Ministério da Cultura estabeleceu as 52 metas do Plano Nacional de Cultura (PNC), sendo que a primeira delas se refere à implementação do Sistema Nacional de Cultura (SNC), posteriormente incorporado à Constituição Federal (artigo 216-A), e que tem entre seus componentes primordiais justamente a criação e institucionalização das secretarias municipais de cultura:

[Aumento do] número de municípios com acordo de cooperação federativa para desenvolvimento do Sistema Nacional de Cultura assinado e publicado no Diário Oficial da União (DOU) e com seus elementos constituídos e institucionalizados, quais sejam: secretaria municipal de cultura ou órgão equivalente, conselho municipal de política cultural, conferência municipal de cultura, plano municipal de cultura e sistema municipal de financiamento à cultura com existência obrigatória do fundo municipal de cultura, em relação ao total de municípios. ${ }^{6}$

Já em sua meta $\mathrm{n}$ ํ.37, o PNC reafirma a necessidade do avanço na constituição dos órgãos gestores da cultura nos municípios brasileiros, indicando explícita e claramente seu caráter central e indispensável:

\begin{abstract}
Meta 37) $100 \%$ das Unidades da Federação (UF) e $20 \%$ dos municípios, sendo $100 \%$ das capitais e $100 \%$ dos municípios com mais de 500 mil habitantes, com secretarias de cultura exclusivas instaladas:

Esta meta refere-se à criação de secretarias com atuação exclusiva na área da cultura, nas Unidades da Federação, no Distrito Federal e nos municípios. Por "secretaria de cultura exclusiva" entende-se órgão da administração direta com competência exclusiva sobre a cultura, sem abranger outras áreas como turismo, meio ambiente, esporte e educação.
\end{abstract}

\footnotetext{
${ }^{5}$ BRASIL. Lei no.12.343, de 02 de dezembro de 2010. Institui o Plano Nacional de Cultura- PNC, cria o Sistema Nacional de Informações e Indicadores Culturais - SNIIC e dá outras providências. Disponível em: https://www.planalto.gov.br/ccivil 03/ ato2007-2010/2010/lei//12343.htm. Acesso em: 04 de janeiro de 2017.

${ }^{6}$ BRASIL. Ministério da Cultura. Portaria no. 123, de 13 de dezembro de 2011. Estabelece as metas do Plano Nacional de Cultura - PNC. Disponível em: http://pesquisa.in.gov.br/imprensa/jsp/visualiza/index.jsp?data=14/12/2011\&jornal=1\&pagina =12 \&totalArquivos=192. Acesso em: 10 de janeiro de 2017.
} 
A construção de políticas de cultura pactuadas entre os entes federados, com participação da sociedade civil, demanda instituições politicamente fortalecidas, profissionalmente qualificadas e focadas na tarefa de contribuir para o avanço da política cultural no Brasil. ${ }^{7}$

O Sistema Nacional de Cultura (SNC) pode ser definido como um instrumento de gestão compartilhada de políticas públicas de cultura entre os entes federados e a sociedade civil, por meio da institucionalização e ampliação da participação social, com vistas ao desenvolvimento humano, social e econômico, com pleno exercício dos direitos culturais e amplo e irrestrito acesso aos bens e serviços culturais. Trata-se de um instrumento em processo de sedimentação que, dentre outros intentos, objetiva quebrar a negativa tradição de descontinuidade da construção e funcionamento das políticas públicas de cultura, o que hoje, após a extinção do MinC e diluição da gestão nacional de cultura no Ministério da Cidadania, ganha uma notória dimensão de incerteza, com possíveis reflexos sobre os órgãos gestores municipais e estaduais.

Como destaca a pesquisadora Luana Vilutis, ainda sobre o período anterior ao desaparecimento do MinC,

\begin{abstract}
$\mathrm{Na}$ atualidade, o alinhamento entre entes federados tem motivações diversas e abrange vários campos da vida em sociedade. Na área cultural do Brasil, podemos pensar também que a escolha de uma organização sistêmica teve uma motivação de proteção, não contra invasões aos territórios, mas fundamentalmente de proteção contra a descontinuidade administrativa da gestão de políticas culturais; uma proteção contra a falta de recursos financeiros e orçamentários para a execução dessas políticas. ${ }^{8}$
\end{abstract}

\title{
O Caso de Ponte Nova
}

A integração de Ponte Nova ao SNC, possibilitou a construção de seu próprio Sistema Municipal de Cultura (SMC), instituído pela Lei Municipal nº. 3.832, de 28 de fevereiro de 2014, sistema que permite o engendramento e a articulação de diferentes elementos dinamizadores das políticas públicas de

\footnotetext{
${ }^{7}$ Idem, ibidem.

${ }^{8}$ VILUTIS, Luana. Op. cit., p.122.
} 
cultura, a saber: coordenação (Secretaria Municipal de Cultura e Turismo); instância de articulação, pactuação e deliberação (Conselho Municipal de Política Cultural, Conferência Municipal de Cultura); instrumentos de gestão (Plano Municipal de Cultura, Sistema Municipal de Financiamento à Cultura e - Sistema Municipal de Informações e Indicadores Culturais). ${ }^{9}$ Seguindo as diretrizes apresentadas pela legislação federal, o SMC é coordenado e gestado pela Secretaria Municipal de Cultura e Turismo, que atende plenamente aos requisitos de autonomia administrativa e orçamentária em relação a outros órgãos componentes da administração pública municipal.

Sob a coordenação e gestão da secretaria, políticas públicas organicamente ligadas aos elementos constituintes do SMC se apresentaram, entre os anos de 2014 e 2016, como realidade e atenderam aos agentes culturais da cidade, especialmente o Sistema Municipal de Financiamento à Cultura (SMFC), que desde o primeiro edital, lançado em 2014, contemplou 12 projetos (o edital de 2016 possuiu ainda 06 projetos suplentes), contando com recursos devidamente salvaguardados no orçamento da pasta, especialmente direcionados para o Fundo Municipal de Cultura.

Necessário dizer ainda que todo processo de seleção e mesmo organização dos editais do SMFC foi organizado em conjunto e com o crivo do Conselho Municipal de Política Cultural (CMPC), instância de articulação, pactuação e deliberação do SMC, composto por representações paritárias do poder público e da sociedade civil, sendo que os representantes desta foram/são democraticamente eleitos dentre os seguimentos artístico-culturais do município, conforme determina a legislação.

Ainda dentro da perspectiva do SMC, há o Sistema Municipal de Informações e Indicadores Culturais (SMIIC), incorporado ao SMC através da Lei Municipal no.4.078, de 5 de dezembro de 2016, porém já incorporado, por exemplo, ao processo de seleção do SMFC desde 2014. O SMIIC se constitui

\footnotetext{
${ }^{9}$ PONTE NOVA. Lei no. 3.832, de 28 de fevereiro de 2014. Dispõe sobre o Sistema Municipal de Cultura de Ponte Nova, seus princípios, objetivos, estrutura, organização, gestão, inter-relações entre os seus componentes, recursos humanos e financiamento e dá outras providências. Disponível em: http://sapl.pontenova.mg.leg.br/sapl documentos/norma juridica/746 texto integral. Acesso em: 28 de dezembro de 2016.
} 
como um banco de dados "referentes a bens, serviços, infraestrutura, investimentos, produção, acesso, consumo, agentes, programas, instituições e gestão cultural, entre outros", tendo entre seus objetivos:

\begin{abstract}
coletar, sistematizar e interpretar dados, fornecer metodologias e estabelecer parâmetros à mensuração da atividade do campo e das necessidades sociais por cultura, que permitam a formulação, monitoramento, gestão e avaliação das políticas de cultura e das políticas culturais em geral, verificando e racionalizando a implementação do Plano Municipal de Cultura - PMC e sua revisão nos prazos previstos. ${ }^{10}$
\end{abstract}

Como sinalizado no parágrafo anterior, o SMIIC já havia sido utilizado como parte do processo de seleção de projetos culturais do Sistema de Financiamento, sendo que apenas foram aceitas inscrições de agentes e movimentos culturais devidamente registrados na plataforma, o que permite a atualização e ampliação das informações sobre as atividades culturais desenvolvidas no âmbito do Município.

\title{
As Políticas Públicas de Cultura e as Artes Cênicas em Ponte Nova
}

A implantação do Sistema Municipal de Indicadores e Informações Culturais foi importante para catalogar os mais diversos grupos e artistas de Teatro da cidade fazendo com que tais informações pudessem marcar a presença da cidade no livro "Memória do Teatro de Grupo: O Teatro em Minas Gerais", organizado por Simone Lara. ${ }^{11}$

Este Sistema se tornou útil à gestão pública de cultura para que pudesse iniciar o traçado de um perfil dos grupos e artistas da cena de Ponte Nova. Estes mesmos grupos e artistas passaram a ocupar cadeiras junto ao Conselho Municipal de Política Cultural, representando o segmento das Artes

\footnotetext{
${ }^{10}$ PONTE NOVA. Lei no. 4.078, de 5 de dezembro de 2016. Altera a Lei Municipal no 3.832/2014, que dispõe sobre o Sistema Municipal de Cultura de Ponte Nova, seus princípios, objetivos, estrutura, organização, gestão, inter-relações entre os seus componentes, recursos humanos e financiamento e dá outras providências. Disponível em: Acesso em: 28 de dezembro de 2016. ${ }^{11}$ LARA, Simone. Memória do teatro de grupo: o teatro em Minas Gerais. Belo Horizonte: Tradição Planalto, 2017.
} 
Cênicas e, como tal, discutindo a aplicação do recurso público em eventos oficiais, em eventos da classe e no estabelecimento, por exemplo, do próprio FESTPON junto ao calendário oficial de eventos do Município. Percebeu-se que a presença dos agentes culturais, em específico das Artes Cênicas, eleitos via chamada pública para compor o referido Conselho, promoveu melhor entendimento da legislação pública de cultura e o empoderamento dos mesmos frente à movimentação do fazer teatral em diálogo com a Secretaria Municipal de Cultura e Turismo, sem que se perdesse a autonomia de suas produções, mas entendendo que a parceria junto à esfera pública foi capaz de ampliar e profissionalizar eventos e ações da área teatral, ampliando o alcance das ações, formando espectadores e estabelecendo processos de mediação teatral.

Participar dos editais públicos de financiamento à cultura trouxe a necessidade de profissionalização dos grupos para poderem inclusive ampliar a atuação dos mesmos e a sua própria manutenção, entendendo que é possível pleitear o recurso público, mas é preciso criar organizações burocráticas e conceituais para profissionalizar o setor, mesmo em cidades interioranas, sem perder a qualidade artesanal e sem sofrer interferência em sua estética de trabalho pelo diálogo com o poder público.

Os grupos de Teatro participantes destas ações aqui citadas pautaram a discussão, movimentaram o surgimento de políticas públicas, analisaram a melhor forma de acesso e garantia de existência do recurso público ser utilizado junto à área, pautando, inclusive, a criação de projetos de Oficinas de Teatro, junto à própria Secretaria de Cultura e Turismo, descentralizando o fazer teatral em projetos desenvolvidos em diferentes Escolas e Bairros, a partir da cessão de espaços para ensaios e produção de cenários e figurinos.

Assim, tem-se que as políticas públicas de cultura são extremamente necessárias para o desenvolvimento sociocultural, principalmente para atender demandas de cidades e regiões fora de eixos culturais aclamados pela mídia ou historicamente e notoriamente mais atendidos por recursos de ordem federal ou estadual. É possível também que estas políticas públicas para os produtores das Artes Cênicas não criem engessamentos e sim possibilidades de diálogo e transformação. No caso de Ponte Nova, as 
políticas públicas de cultura geraram, no período aqui abordado e em específico para a área de Teatro, um caráter maior de profissionalização que vai desde a produção e logística, que envolvem tanto a criação dos espetáculos e projetos, quanto a forma como estes produtores teatrais pleiteiam apoio/patrocínio junto ao poder público e à iniciativa privada.

Questões relacionadas à prestação de contas e ações de publicidade/visibilidade, por exemplo, constantes nos Editais do Sistema Municipal de Financiamento a Cultura de Ponte Nova, foram incorporados à forma de trabalho dos grupos teatrais locais, estabelecendo uma nova via de incentivo para a montagem de projetos a fim de garantir participação no referido Edital. Isso fez com que vários grupos e coletivos começassem a participar não apenas do edital municipal, mas que passassem a se preparar para pleitear também os recursos ofertados a partir dos editais estaduais e federais, buscando recursos e estabelecendo a manutenção de suas produções.

As suas iniciativas particulares, construídas especialmente junto à iniciativa privada, continuaram a existir fazendo com que também grupos e coletivos não dependessem somente dos recursos públicos, uma vez que a profissionalização da cultura proposta pelo poder público despertou a ampliação do olhar do comércio e empresas locais para o entendimento da importância de associar sua marca a projetos culturais. Nesta via, o Teatro em Ponte Nova agregou parceiros importantes e fiéis em suas diversas produções e mesmo para a manutenção de seus espaços, por exemplo.

Os grupos de Teatro, especificamente junto à Política Municipal de Cultura estabelecida em Ponte Nova no período de 2013 a 2016, pautaram importantes demandas e discussões para a implantação do Sistema Municipal de Cultura de Ponte Nova, não só como agentes propositores, mas como articuladores e mobilizadores para que outros segmentos artísticos também interagissem com tal processo, entendendo ser este um fazer político. Num giro de $360^{\circ}$ graus, notou-se que as Artes Cênicas em Ponte Nova, área historicamente tão potente e atuante, se integrou mais aos seus parceiros e carregou o processo de aprendizagem no campo das políticas públicas para a vida diária. 
Em 2017, com a chegada de uma nova gestão municipal, houve a indicação de que se poderia extinguir a Secretaria Municipal de Cultura e Turismo em Ponte Nova, fato este que teve, junto aos artistas de Teatro da cidade, enorme mobilização contrária, indicando-se a necessidade da manutenção da existência do órgão gestor. Foram organizados debates públicos nos quais agentes e movimentos culturais ressaltaram não apenas a necessidade de manutenção da Secretaria, mas das próprias políticas públicas que se encontravam em pleno processo de sedimentação e que poderiam ser colocadas sob ameaça, o que motivou a transformação dos espaços da Arte em locais não apenas da criação lúdica, mas em espaços deliberativos e de governança pública. Ao fim, conseguiu-se, pela força coletiva e democrática, evitar o desaparecimento da Secretaria.

Faz-se necessário o reconhecimento de que as políticas públicas de cultura estão em pleno processo de construção, sedimentação e perenização em Ponte Nova, desde a criação da Secretaria Municipal de Cultura em 2007. Este, no entanto, como anteriormente indicado, não é um fato isolado, mas um processo sistêmico, diretamente ligado ao reconhecimento constitucional da Cultura como força simbólica, econômica e produtora de cidadania.

Hegemonicamente, os mais recentes estudos sobre a gestão pública de cultura, como os já mencionados, indicam como autonomia de gestão, composição de recursos humanos organicamente ligados à área e capacitados, além da existência de infraestrutura própria, são conditio sine qua non para que este processo siga seu percurso de consolidação, de construção coletiva e cidadã.

A também já mencionada recente extinção do MinC e sua transformação em Secretaria Especial de Cultura, vinculada ao Ministério da Cidadania, se deu sob os auspícios de suposta economia, sem que se promovesse amplo e democrático debate ante aos possíveis reflexos negativos para o processo de consolidação das políticas públicas do setor. $O$ vazio programático e as recorrentes ameaças de censura no âmbito de diferentes instituições que compõem o setor, como a ANCINE e a FUNARTE, indicam que, longe de ser "medida de economia", tampouco como um gerenciamento moderno e eficaz, mas em um prejuízo à sociedade, um claro 
e grave retrocesso em relação a toda movimentação e aperfeiçoamento das políticas públicas de cultura nacionais. No esteio dos clamores por economia no serviço público em tempos de crise, cuja natureza não é apenas econômica, mas principalmente político-institucional, aquilo que muitas vezes pode representar a resolução de problemas pode, na verdade causar intensos e dolorosos prejuízos.

Em Ponte Nova, os movimentos culturais, encabeçados por importantes representantes das Artes Cênicas da cidade, e com apoio da imprensa local, conseguiram a manutenção da Secretaria de Cultura e Turismo. Entretanto, desde então, a administração do órgão passou a ser ocupada por gestores que também se ocuparam, simultaneamente, de outras pastas, o que acabou por arrefecer, em grande medida, o processo de ampliação das políticas que, até 2016, seguiam processo de maturação e perenização, especialmente por atrasos e posterior paralização dos editais do Sistema Municipal de Financiamento à Cultura.

Nesta perspectiva não temos, como acontece em muitos casos, o engessamento da produção, no caso teatral, devido a mudanças na área da cultura estabelecidas poder público. No caso, a classe teatral conseguiu, ao menos em parte sustentar, a permanência do órgão gestor da cultura, como elemento estratégico para a ampliação livre e democrática do acesso à cidadania através da cultura, mesmo que este órgão esteja com ação limitada frente a realidade atual da cidade. Não obstante, o fazer teatral se estabelece frente ao poder público pautando, por exemplo, a manutenção do FESTPON, evento que movimenta o fazer cultural da cidade e faz com que a política pública de cultura dialogue com os grupos e coletivos, oportunizando sua manutenção e produção.

Este processo levou ao empoderamento dos agentes culturais, que se apoderaram da política cultural e fizeram com que a mesma fosse pauta de discussão coletiva, democrática e cidadã. O Teatro em Ponte Nova produziu e produz importantes representantes que ocupam o fazer teatral em Minas Gerais e para além dele. Neste sentido, não ignorando o envolvimento de outras manifestações artísticas para a consolidação dos processos de implantação de políticas públicas em Ponte Nova, focamos no segmento das 
As Artes Cênicas e as Políticas Públicas de Cultura em Ponte Nova, Minas Gerais: um breve relato de experiência

Artes Cênicas pela participação ativa que o mesmo vem pautando, dando contorno a várias discussões do fazer cultural antes, durante e depois dos processos institucionais aqui descritos, sem que se a interdite o diálogo com outros atores, como a iniciativa privada, abrindo caminhos para o estabelecimento de um lugar significativo do Teatro enquanto instrumento de mobilização estética, aglutinadora, transformadora e principalmente política. 